\title{
INVERSION OF THE STRUVE TRANSFORM OF HALF INTEGER ORDER
}

\author{
B. H. J. MCKELLAR', M. A. BOX ${ }^{2}$ and E. R. LOVE ${ }^{3}$
}

(Received 18 October 1982)

\begin{abstract}
Defining a spherical Struve function $\mathbf{h}_{n}(t)=\sqrt{\pi / 2 t} \mathbf{H}_{n+1 / 2}(t)$ we show that the Struve transform of half integer order, or spherical Struve transform,

$$
\phi_{n}(x)=\int_{0}^{\infty} h_{n}(x t)(x t)^{-n+1} f(t) d t
$$

where $n$ is a non-negative integer, may under suitable conditions be solved for $f(t)$ :

$$
f(t)=\frac{2}{\pi} \int_{0}^{\infty} h_{n}(x t)(x t)^{n+1}\left(\phi_{n}(x)-\sum_{j=0}^{n} a_{n, j} x^{-2 \jmath}\right) d x,
$$

where $\sum_{j=0}^{n} a_{n, j} x^{-2 J}$ is the sum of the first $n+1$ terms in the asymptotic expansion of $\phi_{n}(x)$ as $x \rightarrow \infty$. The coefficients in the asymptotic expansion are identified as

$$
a_{n, j}=\frac{1}{2^{n-j}(n-j) !} \int_{0}^{\infty}\left(\frac{1}{x} \frac{d}{d x}\right)^{\prime} f(x) d x
$$

It is further shown that functions $\phi_{n}(x)$ which are representable as spherical Struve transforms satisfy $n+1$ integral constraints, which in turn allow the construction of many equivalent inversion formulae.
\end{abstract}

\footnotetext{
'School of Physics, University of Melbourne, Parkville, Victoria 3052 (permanent address) and Theoretical Division, Los Alamos National Laboratory, Los Alamos, New Mexico 87545, U.S.A

${ }^{2}$ School of Physics, University of New South Wales, Kensington, N.S.W. 2033.

${ }^{3}$ Department of Mathematics, University of Melbourne, Parkville, Victoria 3052.

c) Copyright Australian Mathematical Society 1983, Serial-fee code 0334-2700/83.
} 


\section{Introduction}

The standard inversion theorem for the Struve transform, namely that under suitable conditions if

$$
\phi(t)=\int_{0}^{\infty} \mathbf{H}_{\nu}(x t) x f(x) d x
$$

then

$$
f(x)=\int_{0}^{\infty} Y_{\nu}(x t) t \phi(t) d t
$$

was established by Titchmarsh [14] for the case $-\frac{3}{2}<\nu<\frac{1}{2}$. This result was generalised in various ways by Hardy [11], Cooke [6] and Fox [7]. Recently there has been some discussion in the optics literature by Box and McKellar [3] and Fymat [8] of the solution of equation (1) for $f(x)$ for the case $\nu=\frac{3}{2}$; a case which is not covered by any of the above generalisations. Fymat [9] generalised Cooke's result to allow the inversion of (1) for $\frac{1}{2} \leqslant \nu \leqslant \frac{3}{2}$, but his method fails for larger values of $\nu$. It is the purpose of this note to describe the inverse transform to (1) for the case $\nu=n+\frac{1}{2}$ ( $n$ integral and non-negative), and give conditions under which the inversion is valid.

The classical inversion formula (2) is invalid for $\nu=n+\frac{1}{2}$ because $\phi(t)$ defined by (1) does not decrease fast enough as $t \rightarrow \infty$ for it to be possible to represent $f(x)$ as an integral transform of $\phi(t)$. Box and McKellar [2] showed how one can overcome this problem by subtracting certain "badly behaved" terms from $\phi(t)$, leaving a function which is also represented by an integral transform of $f(x)$. This new integral transform can be inverted by standard techniques. The analysis of [2] was purely formal. One aim of this paper is to provide a proof of their result which is expressed as Theorem 1 below. Our proof relies on induction.

In the course of reconciling the different inversion formulae of Box and McKellar [3] and Fymat [8] for the case $\nu=\frac{3}{2}$, Box and McKellar [4] found a set of integral constraints satisfied by the function $\phi$ of (1). They are generalised to $\nu=n+\frac{1}{2}$ in Theorem 2.

Further investigation of the relationship between the inversion formulae of Box and McKellar [3], Fymat [8] and Perelman and Shifrin [13] for the case $\nu=\frac{3}{2}$ led Box and McKellar [5] to yet another inversion formula for this case. This inversion formula is generalised to $\nu=n+\frac{1}{2}$ in Theorem 3. Finally the generalisation of Fymat's inversion formula for values of $v=n+\frac{1}{2}$ for $n \geqslant 1$ is given as Theorem 4.

The existence of a number of apparently different inversion formulae is related to the existence of the integral constraints of Theorem 2. In fact these constraints make it possible to construct a large number of inversion formulae. In any 
numerical application this freedom should be exploited to obtain a rapidly convergent inversion integral.

\section{Statement of results}

Firstly we introduce some definitions. Since we are concerned with Struve functions of half integer order it is convenient to introduce a "spherical Struve function"

$$
\mathbf{h}_{n}(x)=\sqrt{\frac{\pi}{2 x}} \mathbf{H}_{n+\frac{1}{2}}(x)
$$

for $x>0$, by analogy with the familiar spherical Bessel functions ([1], Chapter 10)

$$
j_{n}(x)=\sqrt{\frac{\pi}{2 x}} J_{n+\frac{1}{2}}(x), \quad y_{n}(x)=\sqrt{\frac{\pi}{2 x}} Y_{n+\frac{1}{2}}(x) .
$$

We also introduce the auxiliary functions $f_{j}$ which we define recursively by

$$
f_{j+1}(x)=\frac{1}{x} \frac{d}{d x} f_{j}(x), \quad f_{0}(x)=f(x) .
$$

Our results are then contained in Theorems $1,2,3$ and 4.

THEOREM 1. Let $n$ be a non-negative integer and the functions $f_{f}(x)$ defined in (5) be such that

(i) $f_{,}(x)$ is locally absolutely continuous in $(0, \infty)$ for $0 \leqslant j<n$,

(ii) $f_{f}(x) \rightarrow 0$ as $x \rightarrow \infty$ for $0 \leqslant j<n$,

(iii) $f_{,}(0)=0$ and $f_{j}$ is differentiable at $x=0$ for $0 \leqslant j \leqslant n$,

(iv) $(1+x) f_{j}(x)$ is absolutely integrable on $(0, \infty)$ for $0 \leqslant j \leqslant n$; also let

$$
a_{n, j}=\frac{1}{2^{n-j}(n-j) !} \int_{0}^{\infty} f_{j}(t) d t \text { for } 0 \leqslant j \leqslant n .
$$

Then the Struve transform

$$
\phi_{n}(x)=\int_{0}^{\infty} h_{n}(x t)(x t)^{-n+1} f(t) d t
$$

is inverted by

$$
f(x)=\frac{2}{\pi} \int_{0}^{-\infty} \mathbf{h}_{n}(x t)(x t)^{n+1}\left(\phi_{n}(t)-\sum_{j=0}^{n} \frac{a_{n, J}}{t^{2 j}}\right) d t,
$$

for each $x>0$ at which $f$ is differentiable. Also

$$
\phi_{n}(x)-\sum_{j=0}^{n} \frac{a_{n \cdot j}}{x^{2 j}}=o\left(\frac{1}{x^{2 n}}\right) \text { as } x \rightarrow \infty .
$$


The next theorem shows that, under the conditions of Theorem 1, a Struve transform $\phi_{n}$ given by (7) satisfies $n+1$ integral constraints, namely (10).

THEOREM 2. Under the hypotheses of Theorem 1, including (6) and (7), the relationship

$$
I_{n, r}=\int_{-0}^{\infty} x^{2 r}\left(\phi_{n}(x)-\sum_{j=0}^{r} \frac{a_{n, j}}{x^{2 j}}\right) d x=0
$$

holds for $0 \leqslant r<n$. For $0 \leqslant r=n$ the same equation holds if the upper terminal $\infty$ of the integral is replaced by $\rightarrow \infty$.

A different inversion formula is obtained in the next theorem, with the help of the constraint $I_{n, n}=0$ given in Theorem 2 .

THEOREM 3. Under the hypotheses of Theorem 1, including (6) and (7), and with $y_{n}$ defined as in (4), another inversion formula for (7) with $n$ positive is

$$
\begin{aligned}
f(x)=\frac{2}{\pi} \int_{0}^{-\infty}\{ & \left.y_{n}(x t)+\frac{1}{2 \sqrt{\pi}} \sum_{r=1}^{n} \frac{\Gamma\left(r+\frac{1}{2}\right)}{(n-r) !}\left(\frac{1}{2} x t\right)^{n-2 r-1}\right\}(x t)^{n+1} \\
& \times\left\{\phi_{n}(t)-\sum_{j=0}^{n-1} \frac{a_{n, J}}{t^{2 j}}\right\} d t,
\end{aligned}
$$

for each $x>0$ at which $f$ is differentiable. For $n=0$ (11) is replaced by

$$
f(x)=\frac{2}{\pi} \int_{0}^{-\infty} y_{0}(x t) x t\left\{\phi_{0}(t)-a_{0.0}\right\} d t,
$$

under the same conditions on $x$ and $f$.

The next theorem gives another inversion formula, this time using the constraint $I_{n, n-1}=0$ given in Theorem 2 .

THEOREM 4. Under the hypotheses of Theorem $1, n=0$ being now excluded, the transform (7) is inverted by (11) modified by replacing $\sum_{r=1}^{n}$ by $\sum_{r=2}^{n}$ if $n \geqslant 2$, and by 0 if $n=1$. As in Theorem 3, the inversion formula holds for each $x>0$ at which $f$ is differentiable.

The differentiability condition in our theorems arises from inversion of Fourier cosine transforms by means of Lemma 1 below. It could be replaced by other, less restrictive, conditions arising from more usual inversion theorems, such as [15, Theorems 3 and 6]. However, the more general conditions are more complicated, and we content ourselves with the simple differentiability condition. 


\section{Preliminary lemmas}

LEMMA 1. If $f$ is absolutely integrable on $[0, \infty)$, then for each $x \geqslant 0$ at which $f$ is differentiable,

$$
f(x)=\frac{2}{\pi} \int_{0}^{-\infty} \cos x s d s \int_{0}^{\infty} f(t) \cos s t d t .
$$

Proof. Define $f(x)$ for negative $x$ to make $f$ an even function. Then by [15, page 14, Theorem 4],

$$
f(x)=\frac{1}{\pi} \int_{0}^{+\infty} d s \int_{-\infty}^{\infty} f(t)\{\cos x s \cos s t+\sin x s \sin s t\} d t,
$$

for every $x$ at which $f$ is differentiable. The inner integral can be written as the sum of two integrals, the latter of which vanishes since $f(t)$ sin $s t$ is odd. And since $f(t) \cos s t$ is even, (13) follows.

For $x=0$ this argument may fail, since if $f$ is originally differentiable at 0 it may have unequal one-sided derivatives at 0 when made into an even function. Nevertheless, $[f(y)-f(0)] / y$ is bounded in some interval $-\delta \leqslant y \leqslant \delta$, and this ensures the applicability of the theorem in [15].

The proof of the key Theorem 1 is by induction, so to establish an initial case we first consider $n=0$. Note that this corresponds to $\nu=\frac{1}{2}$ in (1), and is itself outside the range of validity of Titchmarsh's theorem.

LEMMA 2. If $f(x)$ is absolutely integrable on $(0, \infty)$, differentiable at 0 , and $f(0)=0$,

$$
\phi_{0}(t)=\int_{0}^{\infty} h_{0}(x t) x t f(x) d x \text { and } a_{0,0}=\int_{0}^{\infty} f(x) d x,
$$

then

$$
\phi_{0}(t)-a_{0,0} \rightarrow 0 \text { as } t \rightarrow \infty,
$$

and for each $x>0$ at which $f$ is differentiable,

$$
f(x)=\frac{2}{\pi} \int_{0}^{-\infty} h_{0}(x t) x t\left[\phi_{0}(t)-a_{0.0}\right] d t .
$$

Proof. Noting that $h_{0}(x)=x^{-1}(1-\cos x)$, both of the integrals in (14) exist by the absolute integrability of $f(x)$. The first result of the lemma is an immediate consequence of the Riemann-Lebesgue theorem, since

$$
\phi_{0}(x)-a_{0,0}=-\int_{0}^{\infty} f(t) \cos x t d t .
$$


Using (17) in the integral in (16),

$$
\begin{aligned}
\int_{0}^{+\infty} \mathbf{h}_{0}(x s) x s\left\{\phi_{0}(s)-a_{0,0}\right\} d s= & \int_{0}^{+\infty}(\cos x s-1) d s \int_{0}^{\infty} f(t) \cos s t d t \\
= & \int_{0}^{\infty} \cos x s d s \int_{0}^{\infty} f(t) \cos s t d t \\
& -\int_{0}^{-\infty} \cos 0 s d s \int_{0}^{\infty} f(t) \cos s t d t,
\end{aligned}
$$

provided these two repeated integrals separately exist. This is so by Lemma 1 if $f$ is differentiable at $x$; and Lemma 1 also gives

$$
\frac{2}{\pi} \int_{0}^{-\infty} \mathbf{h}_{0}(x s) x s\left\{\phi_{0}(s)-a_{0,0}\right\} d s=f(x)-f(0)=f(x),
$$

completing the proof of Lemma 2.

LEMMA 3. For $\mathbf{h}_{n}$ defined in (3), and $n$ a non-negative integer,

(a)

$$
\mathbf{h}_{n}(x)=\frac{(x / 2)^{n+1}}{(n+1) !}+O\left(x^{n+3}\right) \text { as } x \rightarrow 0,
$$

(b)

$$
\mathbf{h}_{n}(x)=\frac{x^{n-1}}{2^{n} n !}-\frac{\cos (x-n \pi / 2)}{x}+O\left(x^{n-3}\right) \text { as } x \rightarrow \infty .
$$

Proof. Result (a) is an immediate consequence of the power series for $\mathbf{H}_{n}(x)$ ([1], 12.13). Result (b) is obvious in the case $n=0$ from the explicit form of $\mathbf{h}_{0}(x)$. Since for all $n$

$$
\mathbf{h}_{n}(x)=y_{n}(x)+\frac{1}{2 \sqrt{\pi}} \sum_{r=0}^{n} \frac{\Gamma\left(r+\frac{1}{2}\right)}{(n-r) !}\left(\frac{x}{2}\right)^{n-2 r-1},
$$

([10], 8.522(3)), the asymptotic series for the spherical Neumann function $y_{n}$ ([1], 10.1.9) gives, as $x \rightarrow \infty$,

$$
\mathbf{h}_{n}(x)=-\cos \left(x-\frac{1}{2} n \pi\right)+O\left(x^{-2}\right)+\frac{1}{2 \cdot n !}\left(\frac{x}{2}\right)^{n-1}+O\left(x^{n-3}\right) ;
$$

from this (b) follows if $n>0$, since then $n-3 \geqslant-2$.

Lemma 4. Let $f_{m}$ and $f_{m+1}$ be absolutely integrable on $(0, \infty), f_{m}$ be locally absolutely continuous in $(0, \infty)$,

$$
\begin{aligned}
f_{m+1}(t) & =t^{-1} f_{m}^{\prime}(t) \quad \text { almost everywhere, } \\
f_{m}(t)=o\left(t^{-1}\right) \quad \text { as } t & \rightarrow 0, \quad f_{m}(t)=o(t) \quad \text { as } t \rightarrow \infty ;
\end{aligned}
$$


also let

$$
\psi_{n, r}(x)=\int_{0}^{\infty} \mathbf{h}_{n}(x t)(x t)^{-n+1} f_{r}(t) d t
$$

and

$$
b_{n, r}=\frac{1}{2^{n} n !} \int_{0}^{\infty} f_{r}(t) d t,
$$

for $r=m$ and $m+1$, and for each non-negative integer $n$. Then for each such $n$, and positive real $x$,

$$
\psi_{n+1, m}(x)=b_{n+1, m}+x^{-2} \psi_{n, m+1}(x) .
$$

Proof. For fixed positive $x$, Lemma 3 ensures that the integrand in (22) is $O\left(t^{2} f_{r}(t)\right)$ for small $t$ and $O\left(f_{r}(t)\right)$ for large $t$, so that the assumed absolute integrability of $f_{r}$ ensures the existence of $\psi_{n, r}(x)$ for $r=m$ and $r=m+1$.

A recurrence relation for $H_{\nu}(x)$, equation (8) on page 329 of [16], with $\nu=n+\frac{1}{2}$, may be written as

$$
\frac{d}{d x}\left(x^{-n} \mathbf{h}_{n}(x)\right)=\frac{1}{2^{n+1}(n+1) !}-x^{-n} \mathbf{h}_{n+1}(x),
$$

so

$$
\begin{aligned}
\psi_{n+1, m}(x) & =\int_{0}^{\infty}\left\{\frac{1}{2^{n+1}(n+1) !}-\frac{1}{x} \frac{\partial}{\partial t}\left[(x t)^{-n} \mathbf{h}_{n}(x t)\right]\right\} f_{m}(t) d t \\
& =b_{n+1, m}-\frac{1}{x} \int_{0}^{\infty}\left\{\frac{\partial}{\partial t}\left[(x t)^{-n} \mathbf{h}_{n}(x t)\right]\right\} f_{m}(t) d t
\end{aligned}
$$

The existence of the integrals in (26) follows from the absolute integrability hypothesised for $f_{m}$. The assumption that $f_{m}$ is locally absolutely continuous shows that $f_{m}^{\prime}$ exists almost everywhere and allows the integration by parts of the integral in equation (26). Lemma 3 and the assumed behaviour of $f_{m}(x)$ as $x \rightarrow 0$ and $x \rightarrow \infty$ ensure that the integrated terms vanish. Thus

$$
\begin{aligned}
\psi_{n+1, m}(x) & =b_{n+1, m}+\frac{1}{x} \int_{\rightarrow 0}^{-\infty}(x t)^{-n} \mathbf{h}_{n}(x t) f_{m}^{\prime}(t) d t \\
& =b_{n+1, m}+\frac{1}{x^{2}} \int_{-0}^{-\infty}(x t)^{-n+1} \mathbf{h}_{n}(x t) f_{m+1}(t) d t .
\end{aligned}
$$

As the integral in (27) has been shown to be absolutely convergent, (24) follows.

We are now in possession of the results needed to prove our theorems. 


\section{Proof of Theorem 1}

Lemma 2 gives the conclusions (8) and (9) if $n=0$. We therefore suppose that $n \geqslant 1$. Repeated application of Lemma 4 with $m=0,1, \ldots, n-1$ gives

$$
\begin{aligned}
\phi_{n}(x)=\psi_{n, 0}(x) & =b_{n, 0}+\frac{1}{x^{2}} \psi_{n-1,1}(x) \\
& =b_{n, 0}+x^{-2} b_{n-1,1}+x^{-4} \psi_{n-2,2}(x) \\
& =\sum_{j=0}^{n-1} b_{n-j, j} x^{-2 j}+x^{-2 n} \psi_{0, n}(x) .
\end{aligned}
$$

Now $b_{n-\jmath, j}=a_{n, j}$, and

$$
\psi_{0, n}(x)=\int_{0}^{\infty}(1-\cos x t) f_{n}(t) d t=a_{n, n}+o(1)
$$

as $x \rightarrow \infty$ by the Riemann-Lebesgue theorem. Thus

$$
\phi_{n}(x)=\sum_{j=0}^{n} a_{n, j} x^{-2 \jmath}+o\left(x^{-2 n}\right),
$$

proving one result of the theorem, namely (9).

We have in addition from (29) and (30),

$$
\phi_{n}(x)=\sum_{j=0}^{n} a_{n, J} x^{-2 j}-x^{-2 n} \int_{0}^{\infty} f_{n}(t) \cos x t d t,
$$

for all $n$, including $n=0$. We note in passing that the standard results on asymptotic expansion of Fourier integrals permit the construction of further terms in the asymptotic expansion of $\phi_{n}(x)$, should that be desired.

We now proceed to prove (8) by induction. We already know that it holds for $n=0$, by Lemma 2, so we may continue to assume that $n \geqslant 1$. The hypotheses of the theorem as stated imply the corresponding hypotheses with $n$ replaced by $n-1$, and we assume the latter case of the theorem to hold. By this case (8) holds with $n$ replaced by $n-1$, and with (32) it implies

$$
-\frac{1}{2} \pi f(x)=\int_{0}^{-\infty} \mathbf{h}_{n-1}(x s)(x s)^{n} s^{-2 n+2} d s \int_{0}^{\infty} f_{n-1}(t) \cos s t d t,
$$

for each $x>0$ at which $f(x)$ is differentiable.

Hypothesis (i) of Theorem 1 allows the integration of the inner integral in (33) by parts, (ii) and (iii) show that the integrated terms vanish, and (iv) shows that the resulting integral is absolutely convergent. Thus

$$
-s \int_{0}^{\infty} f_{n-1}(t) \cos s t d t=\int_{0}^{\infty} t f_{n}(t) \sin s t d t .
$$


Integration of this equation over $(0, u)$ gives

$$
\chi(u) \equiv-\int_{0}^{u} s d s \int_{0}^{\infty} f_{n-1}(t) \cos s t d t=\int_{0}^{u} d s \int_{0}^{\infty} t f_{n}(t) \sin s t d t .
$$

Since $t f_{n}(t)$ is absolutely integrable by (iv), the double integral on the right side of (35) converges absolutely, and the order of integration may be changed, giving

$$
\begin{aligned}
\chi(u) & =\int_{0}^{\infty} t f_{n}(t) d t \int_{0}^{u} \sin s t d s=\int_{0}^{\infty} f_{n}(t)(1-\cos u t) d t \\
& =\int_{0}^{\infty} f_{n}(t) d t-\int_{0}^{\infty} f_{n}(t) \cos t u d t,
\end{aligned}
$$

since (iv) allows the separation of the integrals in the last step. The outer integration in (35) is permissible because the left side of (34) is a continuous function of $s$; this follows from

$$
\begin{aligned}
\left|\int_{0}^{\infty} f_{n-1}(t)[\cos s t-\cos (s+h) t] d t\right| & =\left|2 \int_{0}^{\infty} f_{n-1}(t) \sin \left(s+\frac{1}{2} h\right) t \sin \frac{1}{2} h t d t\right| \\
& \leqslant|h| \int_{0}^{\infty} t\left|f_{n-1}(t)\right| d t
\end{aligned}
$$

which $\rightarrow 0$ as $h \rightarrow 0$, using (iv). This continuity also permits differentiation of (35), giving

$$
\chi^{\prime}(s)=-s \int_{0}^{\infty} f_{n-1}(t) \cos s t d t .
$$

Thus $\chi(s)$ has a continuous derivative in $s \geqslant 0$; and so also has $s^{-n+1} h_{n-1}(x s)$, by (25) and Lemma 3 . These facts together permit the following integration by parts of the outer integral in (33):

$$
\begin{aligned}
\frac{\pi}{2} f(x)= & x^{n} \int_{0}^{-\infty} \mathbf{h}_{n-1}(x s) s^{-n+1} \chi^{\prime}(s) d s \\
= & x^{2 n-1}\left[\mathbf{h}_{n-1}(x s)(x s)^{-n+1} \chi(s)\right]_{0}^{-\infty} \\
& -x^{2 n-1} \int_{0}^{-\infty} \frac{\partial}{\partial s}\left[\mathbf{h}_{n-1}(x s)(x s)^{-n+1}\right] \chi(s) d s .
\end{aligned}
$$

Using (36), the integrated terms vanish by Lemma 3 and the Riemann-Lebesgue theorem with (iv). Using (36) and (25),

$$
\begin{aligned}
\frac{\pi}{2} f(x)= & -x^{2 n-1} \int_{0}^{-\infty} \frac{\partial}{\partial s}\left[h_{n-1}(x s)(x s)^{-n+1}\right] d s \int_{0}^{\infty} f_{n}(t) d t \\
& +x^{2 n} \int_{0}^{-\infty}\left[\frac{1}{2^{n} n !}-\mathbf{h}_{n}(x s)(x s)^{-n+1}\right] d s \int_{0}^{\infty} f_{n}(t) \cos s t d t .
\end{aligned}
$$

The first double integral is the product of two convergent integrals, one of which vanishes on using Lemma 3; its existence justifies the separation into two double 
integrals in (39). We may split the second double integral into two parts to get

$$
\begin{aligned}
\frac{\pi}{2} f(x)= & \frac{x^{2 n}}{2^{n} n !} \int_{0}^{+\infty} \cos 0 s d s \int_{0}^{\infty} f_{n}(t) \cos s t d t \\
& -\int_{0}^{\rightarrow \infty}(x s)^{n+1} h_{n}(x s) s^{-2 n} d s \int_{0}^{\infty} f_{n}(t) \cos s t d t .
\end{aligned}
$$

The splitting is valid because the first of the double integrals converges by Lemma 1. In fact it is a multiple of $f_{n}(0)$ which vanishes by (iii). Again using (32) we finally obtain

$$
f(x)=\frac{2}{\pi} \int_{0}^{-\infty}(x s)^{n+1} h_{n}(x s)\left(\phi_{n}(s)-\sum_{j=0}^{n} \frac{a_{n, J}}{s^{2 j}}\right) d s .
$$

This completes the proof of Theorem 1 by induction.

\section{Proof of Theorem 2}

Let $J_{n, r}$ denote the integral in (10) modified by replacing the upper terminal by $\rightarrow \infty$. For the case $r=n$ we deduce the modified (10) from (32), including the existence of $J_{n, n}$, as follows.

$$
\begin{aligned}
J_{n, n} & =\int_{0}^{+\infty} x^{2 n}\left(\phi_{n}(x)-\sum_{j=0}^{n} \frac{a_{n, j}}{x^{2 \jmath}}\right) d x \\
& =-\int_{0}^{+\infty} d x \int_{0}^{\infty} f_{n}(t) \cos x t d t \\
& =-\int_{0}^{-\infty} \cos 0 x d x \int_{0}^{\infty} f_{n}(t) \cos x t d t=-\frac{\pi}{2} f_{n}(0)=0,
\end{aligned}
$$

using Lemma 1 and hypotheses (iii) and (iv) in the last line of Theorem 1.

The cases $0 \leqslant r<n$ may be proved by making $r+1$ applications of Lemma 4 to $\phi_{n}(x)=\psi_{n, 0}(x)$, as in the steps from (28) to (29), to obtain

$$
x^{2 r}\left(\phi_{n}(x)-\sum_{j=0}^{r} a_{n, j} x^{-2 \jmath}\right)=x^{-2} \psi_{n-r-1, r+1}(x),
$$

for $0 \leqslant r<n$. So, if $J_{n, r}$ exists,

$$
\begin{aligned}
J_{n, r} & =\int_{0}^{-\infty} x^{-2} \psi_{n-r-1, r+1}(x) d x \\
& =\int_{0}^{-\infty} x^{-2} d x \int_{0}^{\infty} h_{n-r-1}(x t)(x t)^{-n+r+2} f_{r+1}(t) d t .
\end{aligned}
$$


The double integral

$$
\begin{aligned}
K_{n, r} & =\int_{0}^{\infty} f_{r+1}(t) t^{-n+r+2} d t \int_{0}^{\infty} \mathbf{h}_{n-r-1}(t x) x^{-n+r} d x \\
& =\int_{0}^{\infty} f_{r+1}(t) t d t \int_{0}^{\infty} \mathbf{h}_{n-r-1}(u) u^{-n+r} d u
\end{aligned}
$$

is absolutely convergent, by hypothesis (iv) and Lemma 3 , since $\mathbf{h}_{n-r-1}(u) u^{-n+r}$ is $O(1)$ as $u \rightarrow 0$ and $O\left(u^{-2}\right)$ as $u \rightarrow \infty$. Consequently the order of integration can be changed, and

$$
K_{n, r}=\int_{0}^{\infty} x^{-2} d x \int_{0}^{\infty} h_{n-r-1}(x t)(x t)^{-n+r+2} f_{r+1}(t) d t .
$$

What is more, this double integral is absolutely convergent. Hence so is that in (44). This ensures that $J_{n, r}$ exists, and also that the upper terminal $\rightarrow \infty$ can be replaced by $\infty$. Thus by (46), (44), (43) and (22),

$$
K_{n, r}=J_{n, r}=\int_{0}^{\infty} x^{-2} \psi_{n-r-1, r+1}(x) d x=I_{n, r} .
$$

By hypothesis (iv), followed by (5) and hypotheses (i), (ii) and (iii),

$$
\int_{0}^{\infty} f_{r+1}(t) t d t=\int_{\rightarrow 0}^{-\infty} f_{r+1}(t) t d t=\int_{-0}^{\rightarrow \infty} f_{r}^{\prime}(t) d t=\left[f_{r}(t)\right]_{\rightarrow 0}^{-\infty}=0 .
$$

This, with (45) and (47), shows that $I_{n, r}=0$, completing the proof of Theorem 2.

\section{Proof of Theorem 3}

By Theorem 2 with $r=n \geqslant 0$,

$$
0=\frac{2}{\pi 2^{n} n !} \int_{0}^{-\infty}(x t)^{2 n}\left(\phi_{n}(t)-\sum_{J=0}^{n} \frac{a_{n, J}}{t^{2 \jmath}}\right) d t .
$$

We subtract this from (8), which holds since $f$ is differentiable at $x$. Supposing $n \geqslant 1$ we obtain, rearranging terms,

$$
\begin{aligned}
f(x)= & \frac{2}{\pi} \int_{0}^{-\infty}\left[\mathbf{h}_{n}(x t)-\frac{(x t)^{n-1}}{2^{n} n !}\right](x t)^{n+1}\left(\phi_{n}(t)-\sum_{j=0}^{n-1} \frac{a_{n, J}}{t^{2 J}}\right) d t \\
& -a_{n, n} \frac{2}{\pi} \int_{0}^{-\infty}\left[\mathbf{h}_{n}(x t)-\frac{(x t)^{n-1}}{2^{n} n !}\right](x t)^{n+1} t^{-2 n} d t,
\end{aligned}
$$


provided one of these two integrals exists. Actually the latter exists and is zero, because by (25) it is the same as

$$
\begin{aligned}
-\int_{0}^{-\infty}(x t)^{n-1} \frac{1}{x} \frac{\partial}{\partial t}\left[(x t)^{-n+1} h_{n-1}(x t)\right] x^{n+1} t^{-n+1} d t & \\
& =-x^{2 n-1} \int_{\rightarrow 0}^{\rightarrow \infty} \frac{\partial}{\partial t}\left[(x t)^{-n+1} \mathbf{h}_{n-1}(x t)\right] d t \\
& =-x^{2 n-1}\left[(x t)^{-n+1} h_{n-1}(x t)\right]_{\rightarrow 0}^{\rightarrow \infty},
\end{aligned}
$$

and this vanishes by Lemma 3. Also (20) shows that

$$
\mathbf{h}_{n}(x t)-\frac{(x t)^{n-1}}{2^{n} n !}=y_{n}(x t)+\frac{1}{2 \sqrt{\pi}} \sum_{r=1}^{n} \frac{\Gamma\left(r+\frac{1}{2}\right)}{(n-r) !}\left(\frac{1}{2} x t\right)^{n-2 r-1},
$$

and Theorem 3 for $n \geqslant 1$ then follows from (49), (50) and (51).

If $n=0(49)$ is invalid, because the last integral in it is divergent, its integrand being $-\cos x t$. Instead, the result of subtracting (48) from (8) is now

$$
f(x)=\frac{2}{\pi} \int_{0}^{-\infty}\left[(x t) \mathbf{h}_{0}(x t)-1\right]\left[\phi_{0}(t)-a_{0,0}\right] d t,
$$

which with (20) gives (12).

\section{Proof of Theorem 4}

In Theorem 3, the term $r=1$ in (11) can be separated out if it exists. It is

$$
\frac{2}{\pi} \int_{0}^{\infty \infty} \frac{(x t)^{2(n-1)}}{2^{n-1}(n-1) !}\left(\phi_{n}(t)-\sum_{j=0}^{n-1} \frac{a_{n, j}}{t^{2 j}}\right) d t .
$$

By Theorem 2 with $r=n-1$, this exists and is zero; this proves Theorem 4 .

\section{Concluding remarks}

The existence of the constraints $I_{n, r}=0$ established in Theorem 2 allows the construction of many different inversion formulae, including as special cases those already given in the literature. A further example (given in [5] for the case $n=1$ ) is obtainable from Theorem 1 by subtracting a multiple of $I_{n, n}$; it is

$$
f(x)=\frac{2}{\pi} \int_{0}^{\infty}\left[h_{n}(x t)-\frac{(x t)^{n-1}}{2^{n} n !}\right](x t)^{n+1}\left(\phi_{n}(t)-\sum_{j=0}^{n} \frac{a_{n \cdot J}}{t^{2 \jmath}}\right) d t .
$$


In analytical work it may be unimportant which inversion formula is used, but in numerical work some experimentation with the range of possible formulae to obtain one with good convergence and stability properties would be useful.

Finally, we point out that the same procedure (of subtracting "badly behaved" terms from $\phi(t)$ ) will permit the construction of inversion formulae for (1) in the more general case $\frac{1}{2}<\nu \neq n+\frac{1}{2}$ for integral $n$. In this case the inversion is ultimately reduced to Titchmarsh's original formula (2). An example, for $\nu=1$, has been given by McKellar [12].

\section{Acknowledgments}

B. H. J. McKellar wishes to thank the members of the $T-5$ group at the Los Alamos National Laboratory for their hospitality, and the Australian-American Educational Foundation for the award of a Fulbright Senior Scholar Travel Grant.

\section{References}

[1] M. Abramowitz and I. A. Stegun (eds.), Handbook of mathematical functıons (Dover, New York, 1970).

[2] M. A. Box and B. H. J. McKellar, "A generalised inversion theorem for the Struve transform with application to scattering by polydispersion", Unversity of Melbourne preprint UM-P-77/1I (1977), 9 pages.

[3] M. A. Box and B. H. J. McKellar, "Analytical inversion of multispectral extinction data in the anomalous diffraction approximation", Optics Lett. 3 (1978), $91-93$.

[4] M. A. Box and B. H. J. McKellar, "Relationship between two analytic inversion formulae for multispectral extinction data", Appl. Optıcs 18 (1979), 3599-3601.

[5] M. A. Box and B. H. J. McKellar, "Further relations between analytic inversion formulae for multispectral extinction data", Appl. Optics 20 (1981), 3829-3831.

[6] R. G. Cooke, "The inversion formulae of Hardy and Titchmarsh", Proc. London Math. Soc. (2) 24 (1925), 381-420.

[7] C. Fox, "A generalisation of the Fourier-Bessel transform", Proc. London Math. Soc. (2) 29. (1929), 401-452.

[8] A. L. Fymat, "Analytical inversions in remote sensing of particle size distributions. 1: Multispectral extinctions in the anomalous diffraction approximation", Appl. Optics 17 (1978), 1675-1676.

[9] A. L. Fymat, “A generalisation of Cooke's integral inversion formula with application to remote sensing theory", Appl. Math. Comput. 5 (1979), 23-39.

[10] I. S. Gradshteyn and I. M. Ryzhik, Tables of mtegrals, series and products (Academic, New York, 1980).

[11] G. H. Hardy, "Some formulae in the theory of Bessel functions", Proc. London Math. Soc. (2) 23 (1925), lxi-lxiii. 
[12] B H. J. McKellar, "Light-scattering determination of the size distribution of cylinders: an analytic approximation", J. Opt. Soc. Amer. 72(1982), 671-672.

[13] A. Ya. Perelman and K. S. Shifrin, "Improvements to the spectral transparency method for determinıng particle sıze distribution", Appl. Optics 19 (1980), 1787-1793.

[14] E. C. Titchmarsh, "A pair of inversion formulae", Proc. London Math. Soc. (2) 23 (1923), xxxiv-xxxv.

[15] E. C. Titchmarsh. Theony of Fourier integrals, 2nd ed. (Oxford University Press, Oxford, 1948).

[16] G. N. Watson, A treattse on the theory of Bessel functions, 2nd ed. (Cambridge University Press, Cambridge, 1966). 